\title{
Corticosteroid for IgA Nephropathy: Are They Really Therapeutic?
}

\author{
Yujia Lin ${ }^{a}$ Junya Jia ${ }^{a}$ Yipeng Guo ${ }^{b}$ Dandan $\mathrm{He}^{\mathrm{a}}$ Yaru Zhang ${ }^{\mathrm{a}}$ \\ Fuzhen Wang ${ }^{c}$ Tiekun Yan ${ }^{a}$ Youxia Liu ${ }^{a}$ Shan Lin ${ }^{a}$ \\ ${ }^{a}$ Department of Nephrology, General Hospital of Tianjin Medical University, Tianjin, China; ${ }^{b}$ Department of \\ Epidemiology, Tianjin Public Health Bureau, Tianjin, China; 'Department of Statistics, Fenyang Hospital of Shanxi \\ Province, Fenyang, China
}

\section{Keywords}

IgA nephropathy $\cdot$ Corticosteroid $\cdot$ Randomized

controlled trials · Meta-analysis

\begin{abstract}
Background: IgA nephropathy (IgAN) is a common chronic glomerular disease that, in most patients, slowly progresses to end-stage kidney disease. The therapy with corticosteroid in IgAN is still a worldwide problem that is confusing the clinicians. Methods: MEDLINE, EMBASE, the Cochrane Library, and article reference lists were searched for randomized controlled trials (RCTs) that compared corticosteroids with placebo and any other non-immunosuppressive agents in treating IgAN. Twelve RCTs involving 1,057 patients were included. Results: Overall, we found that steroids had statistically significant effects in preventing the decline in renal function (relative risk $0.42,95 \% \mathrm{Cl} 0.25-0.71, p<0.001$ ) and reducing proteinuria (SMD: $-0.58 \mathrm{~g} /$ day, $95 \% \mathrm{Cl}-0.80$ to $-0.36 \mathrm{~g} /$ day) in patients with IgAN. The association between glucocorticoid and risk of kidney outcome was not modified
\end{abstract}

\section{KARGER}

() 2018 S. Karger AG, Basel

E-Mail karger@karger.com

www.karger.com/ajn by steroids' type (prednisone or methylprednisone), dose ( $\leq 30$ or $>30 \mathrm{mg} /$ day), duration ( $\leq 8$ or $>8$ months), or serum creatinine $(<1.10$ or $\geq 1.10 \mathrm{mg} / \mathrm{dL})$. But steroids increased the risk of side effects such as gastrointestinal and endocrinium symptoms. Conclusion: This study provides the clear beneficial effects of the steroids therapy on the kidney function and proteinuria, although it should be used with caution.

(C) 2018 S. Karger AG, Basel

\section{Background}

IgA nephropathy (IgAN) is the most common form of primary glomerulonephritis worldwide, especially in the Asian-Pacific region [1]. Up to $40 \%$ of clinically affected individuals will eventually progress to end-stage kidney disease (ESKD) within 20 years even with optimal blood pressure control and renin-angiotensin system blockade [2-6], which make the treatment of IgAN a critical area. The role of the auto-antigen/autoantibody immune-complex formation and complement ac- 
tivation in the development of IgAN has supported the use of corticosteroids therapy in the management of progressive IgAN. Nonetheless, therapeutic recommendations of corticosteroids were based only on weak evidence, since the outcome of glucocorticoid therapy for IgAN has been inconclusive and conflicting. Although corticosteroid therapy was initially considered to be not delaying the progression of chronic kidney disease [7], many other randomized controlled trials (RCTs) have suggested that it may contribute to some benefit $[8,9]$ in protecting renal function; at the same time, a reduction in daily proteinuria, with few adverse events, could also occur. Additionally, some new RCTs are published. The Supportive Versus Immunosuppressive Therapy for the Treatment of Progressive IgA Nephropathy (STOP-IgAN) trial [10] published in 2017 found that corticosteroids therapy plus supportive care was not superior to supportive care alone in patients with IgAN in delaying the progression of the kidney. However, this study that had the short follow-up for evaluating the renal function deterioration included patients who were on only $>0.75 \mathrm{~g} /$ day of proteinuria (thus showing slow progress). Moreover, recent data from the Therapeutic Evaluation of Steroids in IgA Nephropathy Study (TESTING) [11] and Targeted-release budesonide versus placebo in patients with IgA nephropathy (NEFIGAN) [12] have provided further evidence on the benefits and side effects of corticosteroid treatment in patients with IgAN. These studies remain the latest and the largest in the world for the steroids therapy of IgAN. Several systematic reviews have evaluated the use of glucocorticoid in IgAN; however, these overviews were tested few years and lacked appropriate safety evaluation [13-15]. Among them, the study of Lv et al. [13] has not reported serious and fatal infections and death. The study of Zhou et al. [14] 8 did not evaluate the safety of steroids. And the side effects of respiratory and gastrointestinal symptoms were not documented in the study of Cheng et al. [15]. Therefore, we present the results of a systematic review summarizing currently available evidence from RCTs pertaining to the effect of glucocorticoids in the treatment of IgAN.

\section{Materials and Methods}

Date Sources, Search Strategy, and Inclusion Criteria

Relevant studies were identified by searching the following data sources: MEDLINE (OVID; from 1950 to September 2017), Embase (from 1970 to September 2017) and the Cochrane Library database (Cochrane Central Register of Active Controlled Trials; no date restriction), and abstracts from scientific meetings. Keywords in our search included IgA glomerulonephritis, IgA nephropathy, immunoglobulin A nephropathy, and Berger disease, steroid, prednisone, glucocorticoids, proteinuria, randomized controlled trials, clinical trials, drug therapy. Trials were considered without language restrictions. To evaluate the effects of steroids on IgAN, a study must include the following criteria: (i) the study was an RCT; (ii) the study compared glucocorticoid agents (any dose, type) versus placebo/no treatment; (iii) the study contained deterioration of renal function; and (iv) the study included adverse events.

Data Extraction and Quality Assessment

Data extraction was performed by 2 investigators (Y.L. and D.H.) independently using the standardized extraction form. If there were any different opinions between them, it would be submitted to the other reviewer (Y.L.) and the procedure would be repeated until the questions were resolved. We evaluated the quality of the studies from the Jadad scale [16-18], which is an established procedure. Its scale assigns 0 or 5 points to each of the following 5 items: (i) with or without randomization; (ii) with or without a double-blind design; (iii) the appropriateness of the randomization methods, if used; (iv) the appropriateness of doubleblinding design, if used; (v) the analysis and reasons for withdrawals and dropouts.

\section{Outcomes}

The outcomes in this study were defined as follows: (i) the primary outcome was kidney outcomes, defined as a composite of ESKD, doubling in serum creatinine or $50 \%$ decreasing or more of estimated glomerular filtration rate (eGFR); (ii) the secondary outcome was daily proteinuria at the end of treatment or during the follow-up time, and (iii) adverse events of treatment.

\section{Statistical Analysis}

Dichotomous outcome data from individual trials were analyzed by using the relative risk (RR) measure. For continuous outcomes of proteinuria, we used the weighted difference in means and their 95\% CI at the end of treatment. We also performed subgroup analysis to assess the potential effect for all results based on the type of control drug and duration of treatment. The random effects model using the DerSimonian-Laird method was employed [19]. Heterogeneity was analyzed using an $I^{2}$ test on N-1 degrees of freedom. Funnel plots and Begg's test were used to probe for publication bias. Two-sided $p$ value $<0.05$ was regarded as statistically significant for all included studies. All statistical analyses were performed using Stata SE 14 and Review Manager 5.3 statistical software for the metaanalysis.

\section{Results}

\section{Trial Flow and Study Characteristics}

Our literature search retrieved 1,840 citations, of which 1,760 were excluded during an initial review. We retrieved the full text for the remaineding 80 articles, and 12 clinical trials $[10-12,20-28]$ eligible for the 

studies. Results of a systematic literature search on corticosteroid therapy in IgAN. CCTR, Cochrane Central Register of Controlled Trials; IgAN, IgA nephropathy; RCTs, randomized controlled trials.
Fig. 1. Identification process for eligible

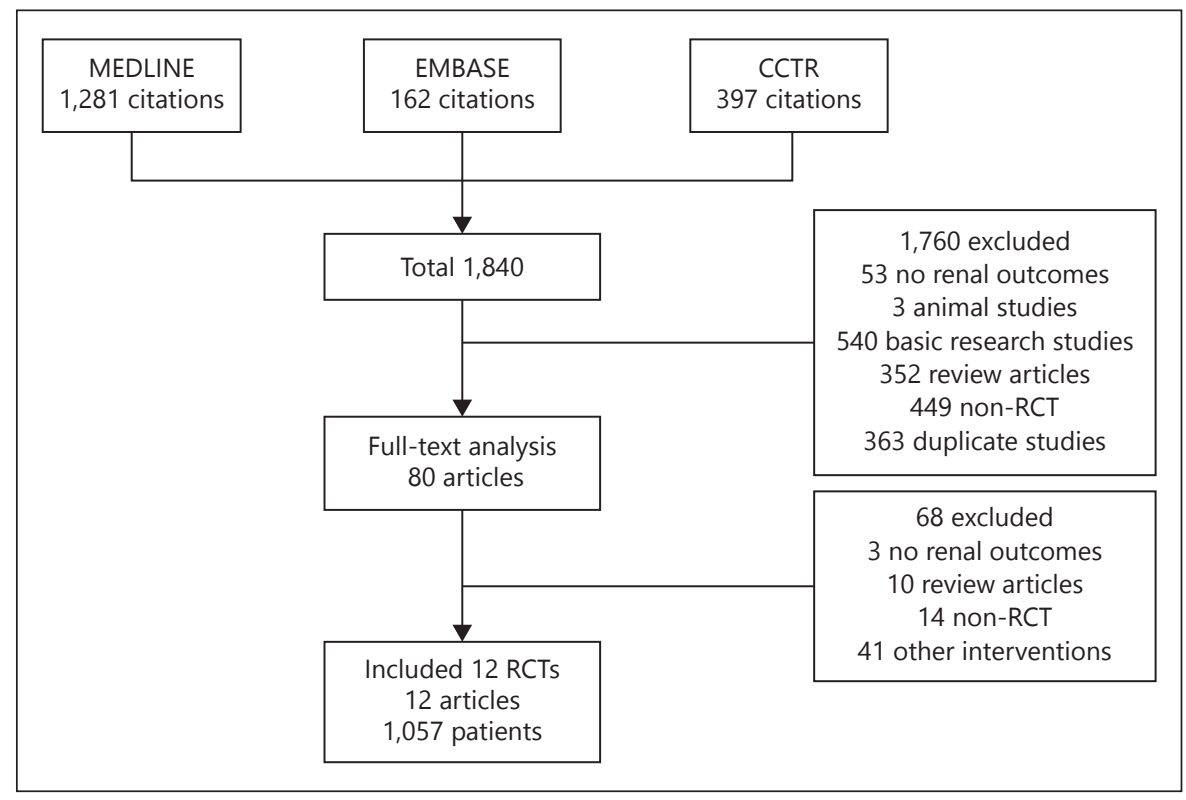

inclusion criteria (Fig. 1). These 12 clinical trials included 1,057 IgAN patients, 560 patients treated with steroid and other 497 patients with control. The follow-up for patients ranged from 12 to 120 months, and the number of patients included in every study ranged from 19 to 261. Pozzi', STOP and TESTING studies were using methylprednisolone, NEFIGAN was using budesonide, and the rest were using prednisone as the choice of steroids. There were 4 studies $[20,23,24,26]$ with a steroid dosage less than $0.5 \mathrm{mg} / \mathrm{kg} /$ day, 5 studies [11, $21,22,27,28]$ with a steroid dosage in the range $0.8 \mathrm{mg} /$ $\mathrm{kg} /$ day to $1 \mathrm{mg} / \mathrm{kg} /$ day, 2 studies $[10,28]$ with a methylprednisolone dosage of $1 \mathrm{~g}$ for 3 days and then tapered prednisone $0.5 \mathrm{mg} / \mathrm{kg}$ every other day, and 1 study [12] with a 8 or $16 \mathrm{mg}$ dosage of targeted-release formulation (TRF)-budesonide. The duration of the treatment ranged from 4 months up to 24 months (Table 1).

\section{Quality Assessment}

A quality assessment of the primary studies is summarized in online supplemental Table S1 (for all online suppl. material, see www.karger.com/doi/10.1159/000489580). The Jadad scores ranged from 0 to 5 points. The study quality on the whole was poor; 2 studies had a Jadad score of 5, 5 studies had a Jadad score of 3, others were less than 3. Participants and investigators were blinded in only 3 trials, 7 studies were founded allocation concealment, and 4 studies satisfy the intention-to-treat analysis criteria.

Corticosteroid for IgAN

\section{Effects on Kidney Outcomes}

Data regarding the effects of glucocorticoids on kidney included ESKD, double Scr, or 50\% decreasing or more of eGFR were available from 10 trials $[11,12,19-26]$ with 859 patients (Figure 2). Among whom 85 events were observed, comprising 23 events in the corticoids group $(n=$ $436,5.28 \%)$ and 62 events in the control group $(n=423$, $14.66 \%)$. Overall, glucocorticoid reduced kidney outcomes by $58 \%$ (RR $0.42,95 \%$ CI $0.25-0.71, p<0.001$ ) in IgAN patients with no evidence of heterogeneity $\left(I^{2}=\right.$ $9.2 \%, p=0.36$ ).

In order to further find the influence of various factors on the incidence of kidney events, we performed subgroup analysis and found that the association between glucocorticoid and risk of kidney outcome was not modified by sample size, age, follow-up time, usage of steroid, steroid dose, type of steroid, steroid duration, using ACEi or ARB in control, baseline proteinuria, serum creatinine, and systolic BP (Fig. 3).

\section{Effects on Proteinuria}

The STOP-IgAN Trial showed corticosteroid monotherapy transiently reduced the protein-to-creatinine ratio at 12 months. However, data on 24 h proteinuria associated with steroid were collected to make a statistical analysis in our paper, not protein-to-creatinine ratio. Six studies [11, 20, 21, 25-27] assessed 24 -h proteinuria in a total of 461 patients, comprising 238 patients in the glucocorticoid treatment group and 223 patients in the control group. Findings suggest that proteinuria at the end 


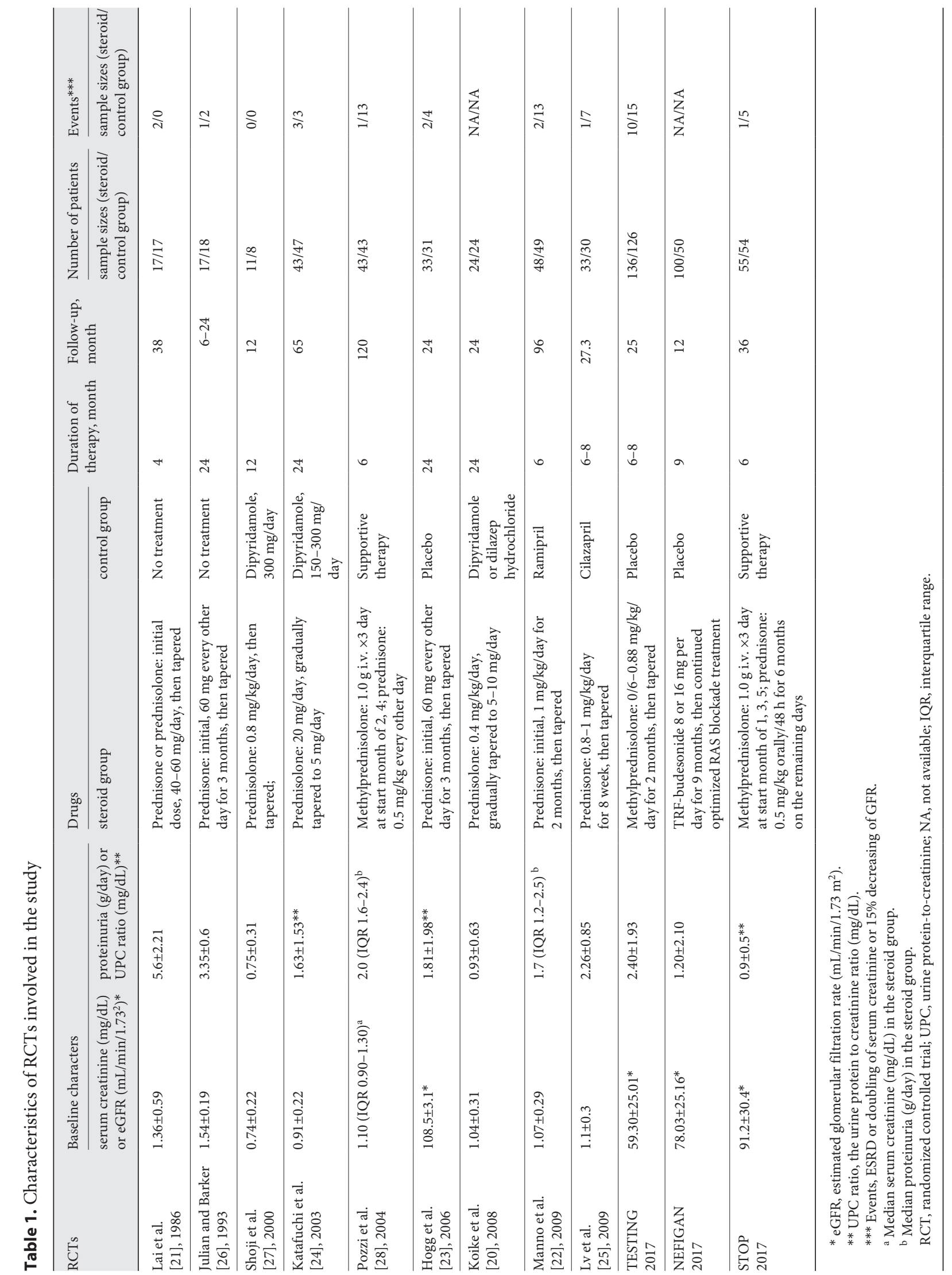




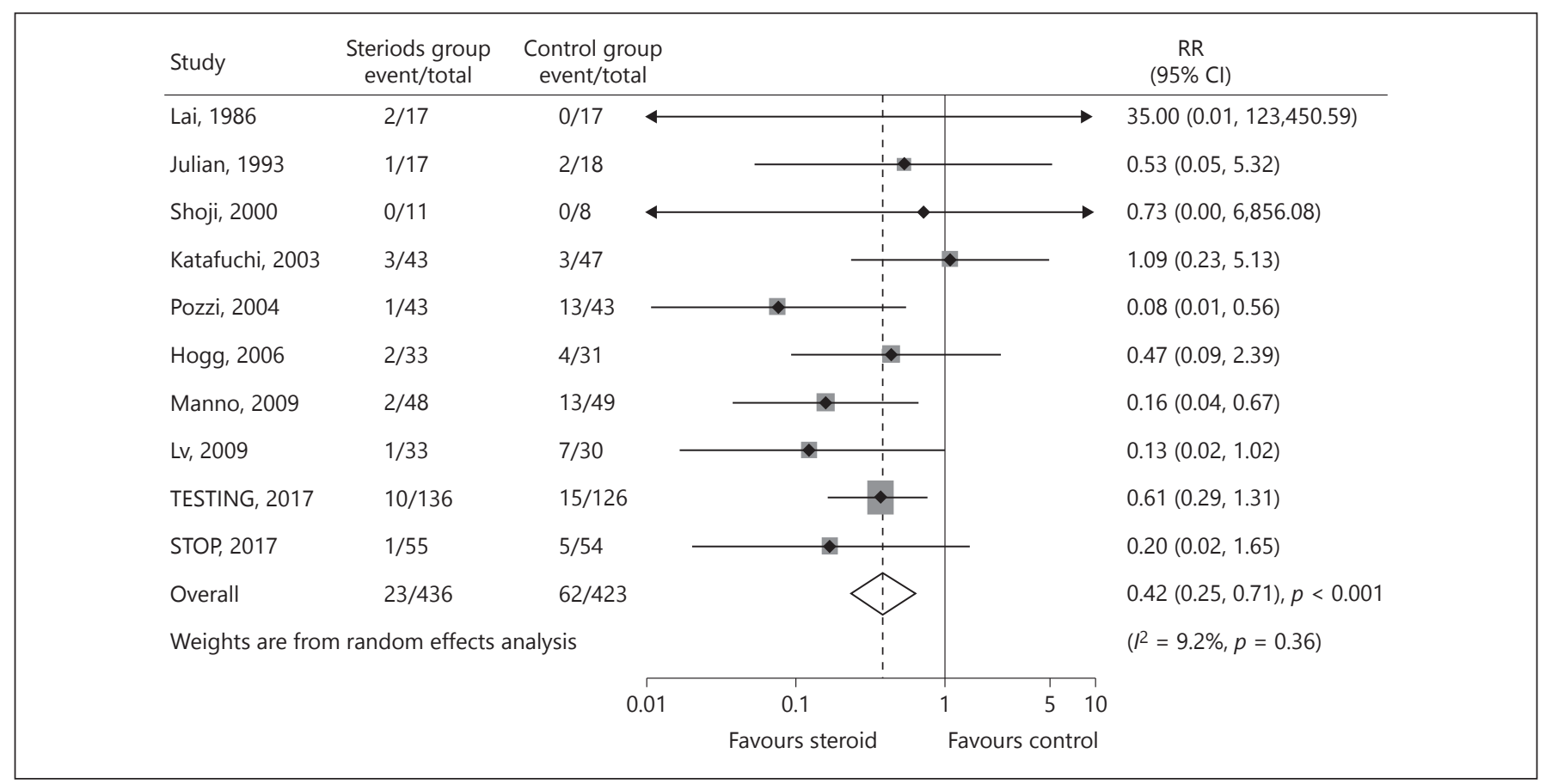

Fig. 2. Effect of steroids on composite renal endpoint (ESRD or doubling of serum creatinine or $50 \%$ decrease of GFR) in patients with IgAN. Boxes and horizontal lines represent RR and 95\% CI, respectively, for each trial. The size of boxes is proportional to the weight of that trial result. Diamonds represent the 95\% CI for

of treatment or during follow-up was significantly lower in steroid group than in the control groups (weighted mean difference, $-0.58 \mathrm{~g} /$ day $[95 \% \mathrm{CI}-0.80$ to $-0.36 \mathrm{~g} /$ day], $p<0.001 ; I^{2}=51 \%, p$ for heterogeneity $=0.07$; Fig. 4).

\section{Adverse Events}

Data on adverse outcomes potentially associated with steroid were collected from 11 RCTs [11, 12, 21-28]. Adverse events such as infections, death, hypertension, cough, insomnia, gastrointestinal bleeding, increased appetite, heartburn, perspiration, palpitations, headache, diabetes mellitus, and cushingoid features reported in the included studies are provided in Table 2. The result showed that steroid therapy was associated with an increased risk for adverse events (RR 1.15, 95\% CI 1.01-1.30; $p=0.03$; Fig. 5). This analysis was dominated by the NEFIGAN study (133 patients; accounting for $48.0 \%$ of the weight). Exclusion of this study did not eliminate the higher risk of adverse events (RR 1.50, 95\% CI 1.12-2.01; $p<0.001)$ associated with steroid therapy but dropped heterogeneity to $31.3 \%$ ( $p=0.16$; online pooled estimates of effect and are centered on pooled RR. Dotted lines on the center of the diamonds represent pooled RR. Solid lines represent that the RR is 1 . GFR, glomerular filtration rate; IgAN, IgA nephropathy; RR, relative risk; ESRD, end-stage renal disease.

suppl. Fig. S1). In all kinds of adverse events, we found that gastrointestinal symptom (RR 1.62, 95\% CI 1.072.47 ) and endocrine symptoms (RR 1.86, 95\% CI $1.23-$ 2.80) contributed more. Other side effects, such as cardiovascular symptoms, respiratory symptoms, endocrine symptoms, neurological symptoms, bonedisorders, infections, and even death in glucocorticoid group had increased tendency, but $p$ value is $>0.05$, which is without any statistical differences.

In order to further find the influence of various factors on the incidence of adverse events, we analyzed related subgroup analysis of side effects. Subgroup analysis results show that the adverse effects cannot be influenced by sample size, age, follow-up time, usage of steroid, type of steroid, steroid dose, steroid duration, using ACEi or ARB in control, baseline proteinuria, serum creatinine, and systolic BP (online suppl. Fig. S2).

\section{Risk of Bias}

The Funnel plots and Begg's test applied to individual trials did not disclose any publication bias $(p=0.93$; online suppl. Fig. S3). 


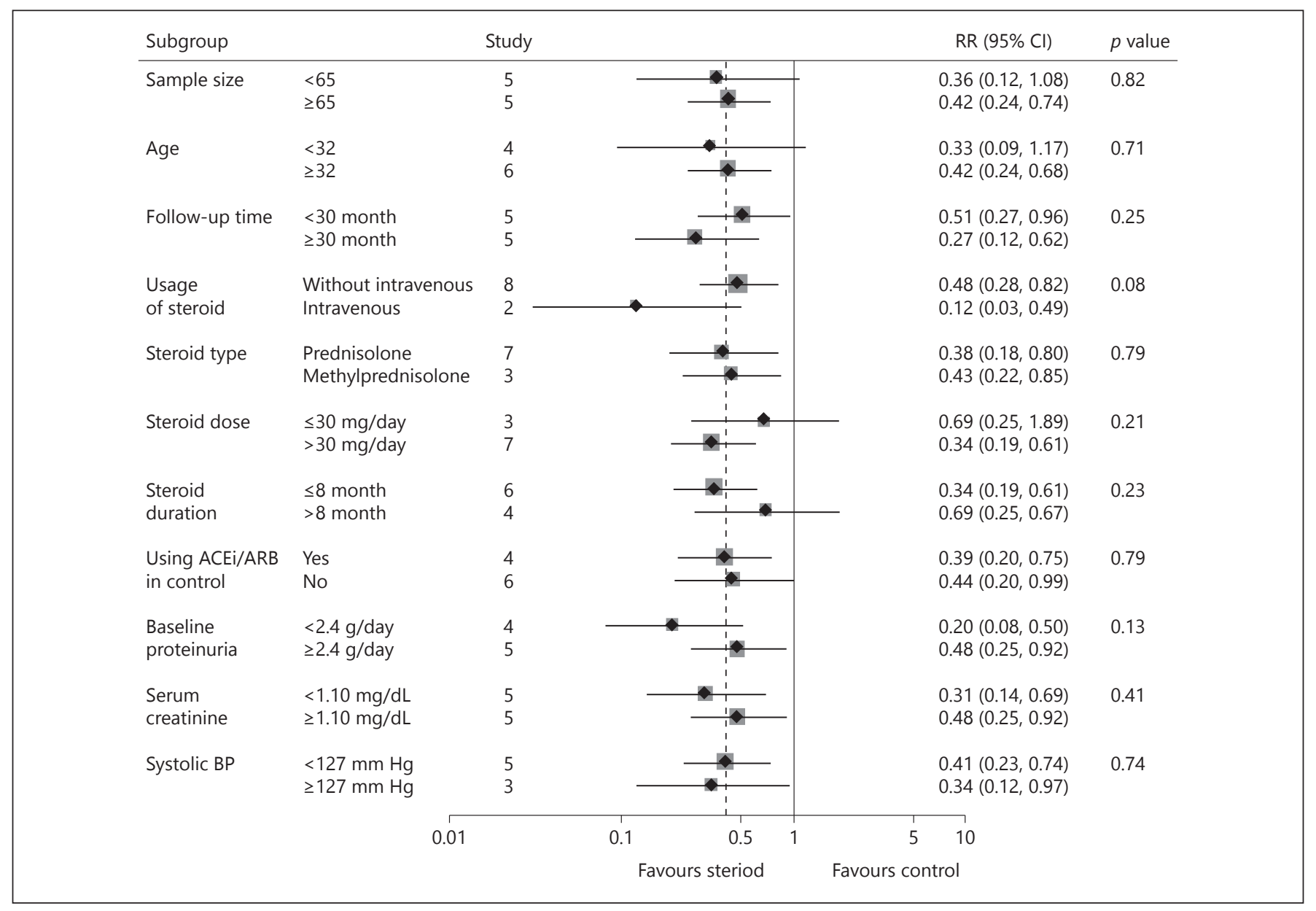

Fig. 3. Subgroup analysis for the effect of corticosteroid on composite renal endpoint (ESRD or 15\% decreasing in serum creatinine or halving of GFR). GFR, glomerular filtration rate; RR, relative risk; ESRD, end-stage renal disease.

\section{Discussion}

The use of steroid is supported by experimental insights into IgAN pathogenesis that suggest an autoimmune component in disease development and progression. Unfortunately, published studies examining the efficacy and safety for corticosteroids therapy in IgAN have reported inconsistent results [21, 24]. In this meta-analysis, quantitative systematic review comprising 12 trials and 1,057 individuals, we have clearly demonstrated that glucocorticoid had statistically significant effects in protecting the renal function and reducing proteinuria in patients with IgAN. On the contrary, it may increase the risk of side effects such as gastroinstestinal symptoms and endocrinium symptoms. The results of this study suggest that glucocorticoid is effective in IgAN patients generally but should be used with caution.
Given that both the pathogenesis and progression of IgAN are linked to defects in mucosal immune regulation and inflammation [29], the use of corticosteroid to prevent kidney failure is well founded. Kidney Disease: Improving Global Outcomes guidelines for glomerulonephritis recommend patients with persistent proteinuria $\geq 1 \mathrm{~g}$ /day, despite 3-6 months of optimized supportive care (including ACE-I or ARBs and blood pressure control), and GFR $>50 \mathrm{~mL} / \mathrm{min} / 1.73 \mathrm{~m}^{2}$, receive a 6 -month course of corticosteroid therapy (prednisone $0.8-1 \mathrm{mg} /$ $\mathrm{kg} /$ day for 2 months and then reduced by $0.2 \mathrm{mg} / \mathrm{kg} /$ day per month for the next 4 months) [30]. The quality of the evidence was generally low (2C). The question of whether corticosteroids exert a benefit if added after optimization of supportive treatment remains unresolved. We defined kidney events as ESKD, double Scr, or 50\% decreasing or more of eGFR which included 10 studies in which 


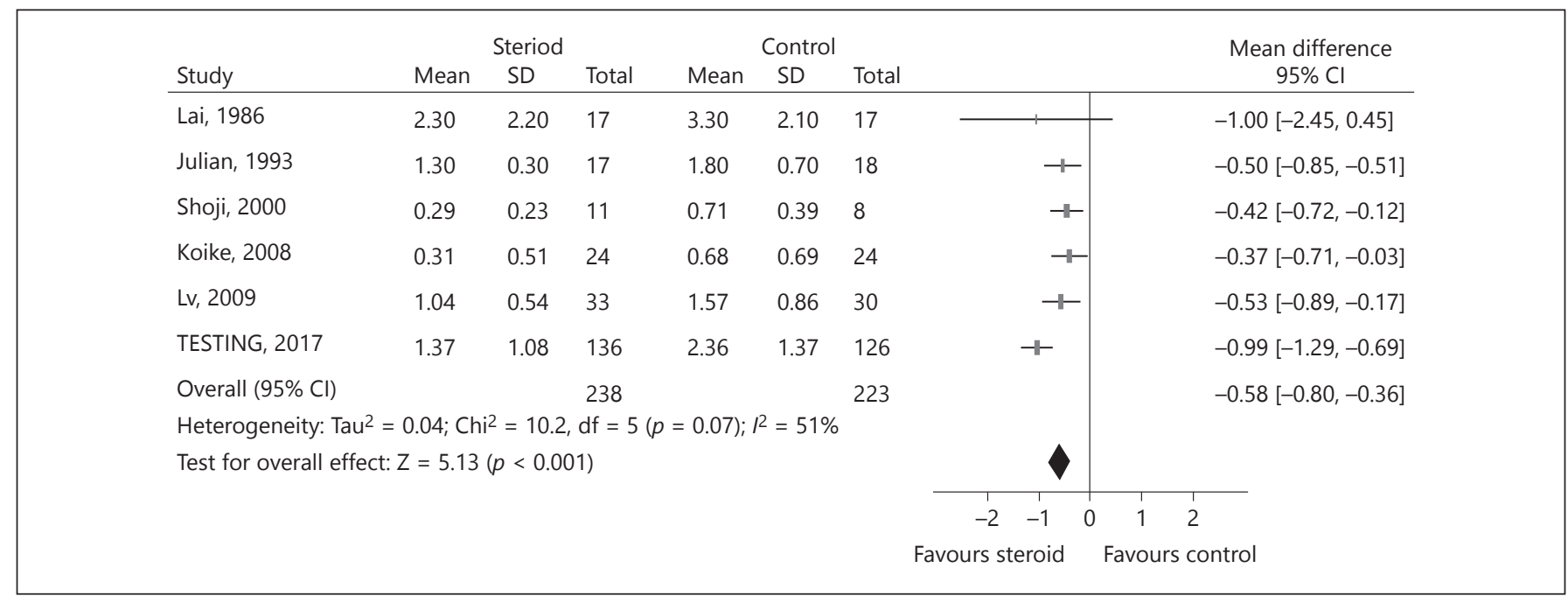

Fig. 4. Effect of steroids on proteinuria in patients with IgAN. Boxes and horizontal lines represent mean difference and 95\% CI, respectively, for each trial. The size of boxes is proportional to the weight of that trial result. Diamonds represent the 95\% CI for pooled estimates of the effect and centered on the pooled mean difference. Dotted line on the center of the diamond represents pooled mean difference. Solid line represents that the mean difference is $0 . \mathrm{IV}$, inverse variance; $\operatorname{IgAN}, \operatorname{IgA}$ nephropathy.

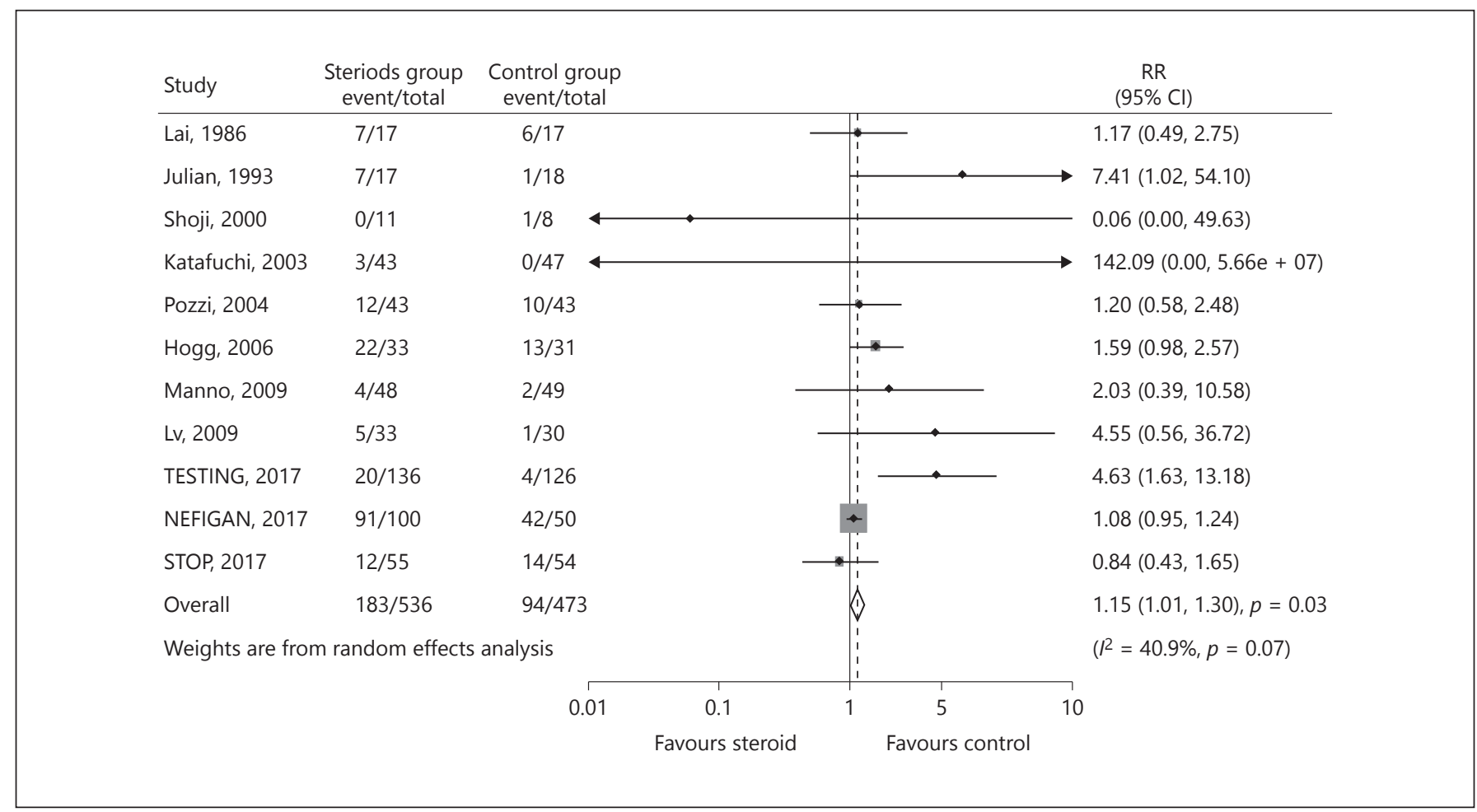

Fig. 5. Adverse events reported in the included trials. Boxes and horizontal lines represent RR and 95\% CI, respectively, for each trial. The size of boxes is proportional to the weight of that trial result. Diamonds represent the $95 \%$ CI for pooled estimates of ef- fect and are centered on pooled RR. Dotted lines on the center of the diamonds represent pooled RR. Solid lines represent that the $R R$ is $1 . R R$, relative risk. 
Table 2. Adverse events reported in the included studies

\begin{tabular}{|c|c|c|c|c|c|}
\hline Adverse events & $\begin{array}{l}\text { Studies } \\
\text { reporting, } n\end{array}$ & $\begin{array}{l}\text { Steroid } \\
\text { group, } n / n\end{array}$ & $\begin{array}{l}\text { Control } \\
\text { group, } n / n\end{array}$ & $\mathrm{RR}(95 \% \mathrm{CI})^{*}$ & $p$ value \\
\hline Total patients with adverse events & 10 & $183 / 536$ & $94 / 473$ & $1.15(1.01-1.30)$ & 0.03 \\
\hline $\begin{array}{l}\text { Specific adverse events } \\
\text { Cardiovascular symptoms (hypertension, } \\
\text { pulmonary embolism, deep vein thrombosis) }\end{array}$ & 6 & $28 / 372$ & $15 / 296$ & $1.11(0.60-2.06)$ & 0.60 \\
\hline $\begin{array}{l}\text { Gastrointestinal symptoms (gastrointestinal } \\
\text { bleeding, increased appetite, heartburn, duodenal } \\
\text { ulcer, gastric perforation, dyspepsia, nausea, } \\
\text { increase of liver enzymes) }\end{array}$ & 5 & $46 / 341$ & $25 / 298$ & $1.62(1.07-2.47)$ & 0.02 \\
\hline $\begin{array}{l}\text { Bone disorders (fracture, osteonecrosis, } \\
\text { avascular necrosis, osteochondroma) }\end{array}$ & 2 & $4 / 191$ & $0 / 180$ & $96.42(0.00-2.21 \mathrm{e}+07)$ & 0.89 \\
\hline Infections & 3 & $20 / 291$ & $5 / 230$ & $1.21(0.42-3.49)$ & 0.73 \\
\hline Death & 2 & $2 / 191$ & $2 / 180$ & $1.64(0.16-17.35)$ & 0.54 \\
\hline
\end{tabular}

859 patients were analyzed to understand the relationship between glucocorticoid treatment and the progression of IgAN. The benefits of corticosteroid for the kidney protection by which steroids reduced the risk of kidney outcomes by $58 \%$ compared with supportive therapy and this finding was confirmed. Subgroup analysis revealed that the association between steroid and risk of kidney outcome was not modified by steroid dose or duration. Some findings indicate that glycan-specific antibodies are associated with the development of IgAN and may represent a disease-specific marker and potential therapeutic target. These findings support a predominant role of an autoimmune mechanism in the pathogenesis of IgAN, which provide strong evidence that steroids may add effectiveness to this autoimmune mechanism. Proteinuria is another important factor to judge the prognosis of glucocorticoid treatment effect in IgAN [31]. So when we analyzed the proteinuria to evaluate the effect of glucocorticoid treatment of IgAN, our research shows that steroids can significantly reduce proteinuria. But it should be noted that steroid therapy can increase the risk of adverse events by $15 \%$. The incidence of gastrointesti- nal symptom, such as gastrointestinal bleeding, increased appetite and heartburn, which are also major side effects, and these side effects increased by $105 \%$ in the steroid group. Also, endocrinium symptoms, including diabetes mellitus/impaired glucose intolerance, acne, hirsutism, and cushingoid features, increased by $86 \%$ in the steroid group. The infection was the most disconcerting side effect; however, few trials reported the incidence of infection even though this was a common adverse event. The recent TESTING trial had showed 11 severe infections (of $136,8.1 \%$ ) in the steroid group and 0 in the placebo group. In the STOP trial [32], 8 patients (of $82,9.8 \%$ ) had severe infection in the corticosteroid group or corticosteroid plus immunosuppressant group. Furthermore, a recent study conducted by Cai et al. [33] showed that of the 369 patients with steroids therapy in IgAN, severe or fatal infection happened in 18 patients (4.9\%). Of all the studies, only TESTING and STOP trials mentioned death, in which 2 cases of the steroid group in TESTING (of 136, $1.5 \%$ ) and 1 in the placebo group (of 126, $0.8 \%$ ) died. In the STOP study, 1 case of the steroid group died in an accident and no deaths occurred in the placebo group. 
These data report a high risk of severe infection or even death in the steroids regimen in IgAN. Whether low-dose steroids may provide less adverse effects in IgAN is still unknown. The ongoing international randomized control trial TESTING study (ClinicalTrials.gov No. 01560052) evaluates the efficacy and safety of lower (methylprednisolone: $0.4 \mathrm{mg} / \mathrm{kg}$ /day per day for 2 months, and then tapered) versus full-dosage (methylprednisolone: $0.8 \mathrm{mg} / \mathrm{kg} /$ day, maximal $48 \mathrm{mg} /$ day, taper by $8 \mathrm{mg} /$ day each month stopped at 6 months) steroids regimens in patients with IgAN. We hope this trial could answer the question of efficacy and safety of different steroids. The NEFIGAN study indicated one hundred and 33 patients experienced adverse events, comprising 91 patients in the TRF-budesonide group (91\%) and 42 patients in the control group (84\%). Though the number of side effects was very high, there were no serious infections and death happened in TRF-budesonide treatment. It has a preferable tolerability profile, which may provide another choice of treatment for IgAN patients [34]. This tolerability still needs to be confirmed in larger studies and more patients. Therefore, we still need to be cautious about using steroid, because many side effects can be triggered by the steroid therapy.

Strengths of this meta-analysis are the large volume of data that was able to be included, and the rigorous methodology used. However, our study also has the following limitations: (1) Existence of potential confounding factors could not be excluded. For example, the control group is not homogeneous, as it consists ACEI/ARBs or antiplatelet drugs, so that different agents might not have the same risk-benefit ratio in IgAN patients; (2) the prognosis evaluation index is less or data are incomplete in this paper; (3) Our involved studies have poor quality assessment. The limitations of the current study mean that large high-quality RCTs are needed to reliably emphasize the efficacy and safety of steroids treatment in IgAN patients.

\section{Conclusion}

In conclusion, this study provides clear beneficial effects of steroid therapy on kidney function in patients with IgAN. It also points out the significant adverse events that occur as a consequence of such therapies. These results suggest that future guidelines should balance the risk and benefit of corticosteroid treatment in IgAN.

\section{Acknowledgment}

This work was supported by grants from the National Natural Science Foundation (81600553, 81270791, and 30800529), Tianjin Municipal Science and Technology Commission Foundation (14JCYBJC27900), The 12th Five-Year Plan National Science and Technology Support Program (No. 2011BAI10B02) and Shanxi Public Health Bureau Foundation (201302051), the General Hospital of Tianjin Medical University Youth Incubation Foundation(ZYYFY2015001). Study design; collection, analysis, and interpretation of data; writing the report; and the decision to submit the report for publication.

\section{Disclosure Statement}

The authors declare that they have no conflicts of interest to disclose.

\section{Financial Disclosure}

The authors declare that they have no other relevant financial interests.

\section{References}

1 Wyatt RJ, Julian BA: IgA nephropathy. N Engl J Med 2013;368:2402-2414.

-2 Coppo R, D’Amico G: Factors predicting progression of IgA nephropathies. J Nephrol 2005; 18:503-512.

3 Berthoux F, et al: Predicting the risk for dialysis or death in IgA nephropathy. J Am Soc Nephrol 2011;22:752-761.

4 Shima Y, et al: Spontaneous remission in children with IgA nephropathy. Pediatr Nephrol 2013;28:71-76.

5 Gutierrez E, et al: Long-term outcomes of IgA nephropathy presenting with minimal or no proteinuria. J Am Soc Nephrol 2012;23:17531760.
6 6 Tang Z, et al: Idiopathic IgA nephropathy with diffuse crescent formation. Am J Nephrol 2002;22:480-486.

7 D'Amico G: Influence of clinical and histological features on actuarial renal survival in adult patients with idiopathic IgA nephropathy, membranous nephropathy, and membranoproliferative glomerulonephritis: survey of the recent literature. Am J Kidney Dis 1992;20:315-323.

8 Galla JH: IgA nephropathy. Kidney Int 1995; 47:377-387.

9 Kobayashi Y, et al: Moderately proteinuric IgA nephropathy: prognostic prediction of individual clinical courses and steroid thera- py in progressive cases. Nephron 1989;53: 250-256.

10 Rauen T, et al: Effects of two immunosuppressive treatment protocols for IgA nephropathy. J Am Soc Nephrol 2018;29:317325.

$11 \mathrm{Lv}$ J, et al: Effect of oral methylprednisolone on clinical outcomes in patients with IgA nephropathy: the TESTING randomized clinical trial. JAMA 2017;318:432-442.

12 Fellstrom BC, et al: Targeted-release budesonide versus placebo in patients with IgA nephropathy (NEFIGAN): a doubleblind, randomised, placebo-controlled phase 2b trial. Lancet 2017;389:2117-2127. 
$13 \mathrm{Lv}$ J, et al: Corticosteroid therapy in IgA nephropathy. J Am Soc Nephrol 2012;23:11081116.

14 Zhou YH, et al: Steroids in the treatment of IgA nephropathy to the improvement of renal survival: a systematic review and meta-analysis. PLoS One 2011;6:e18788

15 Cheng J, et al: Efficacy and safety of glucocorticoids therapy for IgA nephropathy: a meta-analysis of randomized controlled trials. Am J Nephrol 2009;30:315322.

16 Juni, P, Altman DG, Egger M: Systematic reviews in health care: assessing the quality of controlled clinical trials. BMJ 2001;323:4246.

17 Schulz KF, et al: Empirical evidence of bias. Dimensions of methodological quality associated with estimates of treatment effects in controlled trials. JAMA 1995;273:408-412.

18 Jadad AR, et al: Assessing the quality of reports of randomized clinical trials: is blinding necessary? Control Clin Trials 1996;17:1-12.

19 DerSimonian R, Laird N: Meta-analysis in clinical trials. Control Clin Trials 1986;7:177188.

20 Koike M, et al: Clinical assessment of lowdose steroid therapy for patients with IgA nephropathy: a prospective study in a single center. Clin Exp Nephrol 2008;12:250255 .
21 Lai KN, et al: Corticosteroid therapy in IgA nephropathy with nephrotic syndrome: a long-term controlled trial. Clin Nephrol 1986; 26:174-180.

22 Manno C, et al: Randomized controlled clinical trial of corticosteroids plus ACE-inhibitors with long-term follow-up in proteinuric IgA nephropathy. Nephrol Dial Transplant 2009;24:3694-3701.

23 Hogg RJ, et al: Clinical trial to evaluate omega-3 fatty acids and alternate day prednisone in patients with IgA nephropathy: report from the Southwest Pediatric Nephrology Study Group. Clin J Am Soc Nephrol 2006; 1 467-474.

24 Katafuchi R, et al: Controlled, prospective trial of steroid treatment in IgA nephropathy: a limitation of low-dose prednisolone therapy. Am J Kidney Dis 2003;41:972-983.

$25 \mathrm{Lv}$ J, et al: Combination therapy of prednisone and ACE inhibitor versus ACE-inhibitor therapy alone in patients with IgA nephropathy: a randomized controlled trial. Am J Kidney Dis 2009;53:26-32.

26 Julian BA, Barker C: Alternate-day prednisone therapy in IgA nephropathy. Preliminary analysis of a prospective, randomized, controlled trial. Contrib Nephrol 1993;104: 198-206.

27 Shoji T, et al: Early treatment with corticosteroids ameliorates proteinuria, proliferative lesions, and mesangial phenotypic modulation in adult diffuse proliferative IgA nephropathy. Am J Kidney Dis 2000;35:194201

28 Pozzi C, et al: Corticosteroid effectiveness in IgA nephropathy: long-term results of a randomized, controlled trial. J Am Soc Nephrol 2004;15:157-163.

29 Suzuki H, et al: Aberrantly glycosylated IgA1 in IgA nephropathy patients is recognized by IgG antibodies with restricted heterogeneity. J Clin Invest 2009;119:16681677.

30 Radhakrishnan J, et al: The KDIGO practice guideline on glomerulonephritis: reading between the (guide)lines - application to the individual patient. Kidney Int 2012;82:840856.

31 Xie J, et al: Predicting progression of IgA nephropathy: new clinical progression risk score. PLoS One 2012;7:e38904.

- 32 Rauen T, et al: Intensive supportive care plus immunosuppression in IgA nephropathy. N Engl J Med 2015;373:2225-2236.

33 Cai Q, et al: Severe adverse effects associated with corticosteroid treatment in patients with IgA nephropathy. Kidney Int Rep 2017;2: 603-609.

34 Hogg RJ: Idiopathic immunoglobulin A nephropathy in children and adolescents. Pediatr Nephrol 2010;25:823-829. 